\title{
Guest Reviewers 1989
}

The Editorial Board acknowledges, with thanks, the assistance of the following guest reviewers:

Abou-Madi, Mounir / MONTREAL

Ali, M. / LONDON

Ansley, David M. / VanCouver

Archer, D.P. / CALGARY

Atkinson, John W. / OTTAWA

Barry, A. Wayne / OTTAWA

Belisle, Sylvain / MONTRÉAL

Belo, Susan / TORONTo

Bevan, Joan C. / MONTRÉAL

Biehl, Diane R. / WinNiPEG

Bissonnette, Bruno / TORONTO

Blaise, B.A. / MONTREAL

Blanc, Victor / MONTRÉAL

Bond, D.M. / LONDON

Bourke, M. I OTTAWA

Buczko, G. / TORONTO

Burrows, Frederick A. / TORONTO

Butler, P. / KINGSTON

Byrick, R.J. / TORONTO

Calverley, R.K. / SAN DIEGo, CA

Cameron, C. / WINNIPEG

Cannon, J. / WTNmipeg

Cattran, C. / OTTAWA

Cheng, Davy C.H. / TOROnto

Chung, Frances F.T. / TORONTO

Clairoux, Michel / SHERBRoOKE

Code, William / SASKatoON

Couture, Jacques / MONTRÉAL

Cowie, Neil / SAsKatoon

Cox, Robin G. / Calgary

Crone, Lesley-Ann / SASKaTOON

Curran, Michael / OTTAWA

Dolman, John / VANCOUVER
Donati, François / MONTRÉaL

Donen, Neil / WINNIPEG

Douglas, M. Joanne / VANCOUvER

Duke, Peter / WINNIPEG

Dwane, Peter D. / KIngston

Eagle, C.J. / CALGary

Edelist, Gerald / TORONTO

Elliot, R. / OTTAWA

Finegan, B.A. / EDMONTON

Flynn, J. / ST. JOHN'S

Fox, Gordon S. / MONTREAL

Froese, Alison B. / KINGSTON

Gelb, Adrian / LONDON

Goresky, G.V. / CALGaRY

Guest, Stuart / EDMONTON

Ha, H.C. / SASKATOON

Hall, R.I. / HaLIFAX

Halpern, Stephen H. / TORONro

Hamilton, Philip P. I otrawa

Hardy, Jean-Francois / MONTrÉal

Hemmings, Gisele / MONTrÉal

Hew, Ernest M. / TORONTo

Hewson, John R. / HamiLton

Houston, P. / TORONTO

Hudson, R.J. / WINNIPEG

Johnson, G. / OTTAWA

Kamani, Allaudin A. / VANCOUVER

Kehler, Chris H. / WINNIPEG

Keith, Ian / SAINT JOHN

King, F.G. / ST. JOHN'S

Kitts, John B. / OTTAWA

Knill, Richard L. / LONDON

Kolton, M. / TORONTO 
Labrecque, Pascal / MONTRÉAL

Launcelott, Gordon / HALIFAX

Lerman, Jerrold / TORONTO

Lewis, Geraint / OTTAWA

MacAdams, Charles / CALGARY

MacEwan, Peter C. / OTTAwA

MacKenzie, T.A. / TORONTO

McIntyre, John W.R. / EDMONTON

McLean, J. / TORONTo

McMorland, Graham H. / VANCOUVER

Mallon, Joseph S. / roRONTO

Malm, Richard / VANCOUVER

Manninen, Pirjo / LONDON

Martineau, Raymond / OTTAWA

Mazer, C. David/ TORONTO

Mensink, Fred / EDMONTON

Miller, D. / OtTawa

Moore, Anne K. / MONTRÉAL

Moote, Carol A. / Landon

Morrison, D.L. / HaLifax

Morrison, James D. / Halifax

Murkin, J.M. / LONDON

Mutch, W.A.C. / WINNIPEG

Nathan, H.J. / OTTAWA

O'Connor, G. I Vancouver

O'Conner, J.P. / MONTREAL

Ong, B.Y. / WINNIPEG

Oxorn, Donald C. / HaLifax

Papworth, David / SASKatoon

Parsons, David / vancouver

Penning, J. / OtTaWA

Perera, E. / TORONTO

Pietak, S. / KINGSTON
Plourde, Gilles / MONTRÉAL

Quance, Daniel R. / MONTRÉAL

Ralley, Fiona, R. / MONTRÉAL

Ramsay, James G. / MONTREAL

Rayussin, Patrick / LAUSANNE, SWITZERLAND

Redfern, Paul A. / ST. JOHN's

Reid, G. / OtTawa

Rogers, K. / TORONTO

Rosaeg, O.P. / OTTAWA

Rosales, Jose K. / HaLIFAX

Rose, Keith / TORONTO

Scott, W. Andrew C. / MONTRÉAL

Shelley, E.S. / KINGSTON

Simpson, T. / KINGSTON

Slinger, Peter / MONTRÉAL

Smith, J. Bruce / MONTREAL

Spence, Desmond G. / MONTRÉAL

Splinter, W. / OTTAWA

Steward, David J. / VANCoUvER

Sung, Henry M.H. / vancouver

Tetrault, Jean-Pierre / SHERBROOKE

Thistlewood, John / CALGARY

Thomson, Ian R. / WINNIPEG

Townsend, Gary E. / VANCOUVER

Trop, Davy / MONTRÉAL

Vaghadia, Himat / VANCOUVER

Weeks, Sally K. / MONTREAL.

Whalley, David G. / MONTRÉAL

Whiting, Robert / EDMONTON

Williams, R.T. / CALGARY

Writer, W.D.R. / HALIFAX

Zaharia, Francoise / SHERBROOKE 